\title{
Steep rise in norovirus cases and emergence of a new recombinant strain GII.P16-GII.2, Germany, winter 2016
}

S Niendorf ${ }^{12}$, S Jacobsen ${ }^{12}$, M Faber ${ }^{3}$, AM Eis-Hübinger ${ }^{4}$, J Hofmann ${ }^{5}, 0$ Zimmermann ${ }^{6}$, M Höhne $^{12}$, CT Bock ${ }^{1}$

1. Department of Infectious Diseases, Robert Koch-Institute, Berlin, Germany

2. Consultant Laboratory for Noroviruses, Robert Koch-Institute, Berlin, Germany

3. Department for Infectious Disease Epidemiology, Robert Koch-Institute, Berlin, Germany

4. Institute of Virology, University of Bonn Medical Centre, Bonn, Germany

5. Institute of Medical Virology, Charité University Medicine, Berlin, and Labor Berlin, Charité-Vivantes GmbH, Berlin, Germany

6. Institute of Medical Microbiology, Göttingen, Germany

\section{Correspondence: Sandra Niendorf (NiendorfS@rki.de)}

Citation style for this article:

Niendorf S, Jacobsen S, Faber M, Eis-Hübinger AM, Hofmann J, Zimmermann O, Höhne M, Bock CT. Steep rise in norovirus cases and emergence of a new recombinant strain GII.P16-GII.2, Germany, winter 2016. Euro Surveill. 2017;22(4):pii=30447. DOI: http://dx.doi.org/10.2807/1560-7917.ES.2017.22.4.304472

Article submitted on 20 December 2016 / accepted on 25 January 2017 / published on 26 January 2017

Since early November 2016, the number of laboratoryconfirmed norovirus infections reported in Germany has been increasing steeply. Here, we report the detection and genetic characterisation of an emerging norovirus recombinant, GII.P16-GII.2. This strain was frequently identified as the cause of sporadic cases as well as outbreaks in nine federal states of Germany. Our findings suggest that the emergence of GII.P16GII. 2 contributed to rising case numbers of norovirus gastroenteritis in Germany.

In 2016, the increase of notified norovirus cases in the winter season was unexpectedly strong and early (Figure 1) in Germany. In November 2016, 14,872 laboratory-confirmed cases were reported to the national public health authority compared with a median of 7,810 cases in the same month of the previous five years. This may be due to a new variant's ability to escape herd immunity to the previously circulating strains. In this study, we conducted a phylogenetic analysis of the currently circulating norovirus strains in order to assess whether one or several new strains could be responsible for the current steep rise in norovirus cases.

\section{Sample collection and molecular characterisation}

The Consultant Laboratories (CL) and National Reference centre (NRC) are officially appointed and funded by the German Federal Ministry of Health and play a central role in detection and prevention of infection disease in Germany. The coordination of the CL and NRC is hosted by the Robert Koch-Institute. The $\mathrm{CL}$ for norovirus at the Robert Koch-Institute is focused on the molecular surveillance of viral gastroenteritis pathogens, especially noroviruses. For genotyping analysis, stool specimens from norovirus-positive outbreaks were sent to the CL by diagnostic laboratories, physicians and local public health authorities. Between September and December 2016, 240 norovirus positive stool samples from patients with norovirus-associated AGE from 13 federal states of Germany were analysed at the $C L$ for noroviruses. Altogether 175 samples were associated with 69 outbreaks, mainly in childcare facilities $(n=39$ outbreaks) and nursing homes ( $n=12$ outbreaks) in 11 of 16 federal states (Baden-Wuerttemberg, Bavaria, Berlin, Hesse, Lower Saxony, Mecklenburg-Western Pomerania, North Rhine-Westphalia, RhinelandPalatinate, Saxony, Schleswig-Holstein and Thuringia). Altogether 65 samples were from sporadic AGE and were sent by hospitals and diagnostics laboratories from six federal states (Baden-Wuerttemberg, Berlin, Brandenburg, Hamburg, Lower Saxony and North Rhine-Westphalia).

Samples were genotyped as previously described [6] by phylogenetic analysis of ORF1 and ORF2 sequences. To determine the recombination breakpoint, 14 samples of the new norovirus recombinant were analysed in addition, using a newly established semi-nested RT-PCR spanning the 3' end of the ORF1 and the P 2 domain. In brief, RT-PCR reactions were performed using SuperScriptIII One-Step RT-PCR system Platinum TAQ DNA Polymerase (Thermo Fisher, Walthman MA, US) and primer sets NV1a (5'-ATGAATATGAATGAAGATGG-3'), NV1b (5'-ATGAACACAATAGAAGATGG-3'), NV348a (5'-GGTTRACCCARGAATCAAA-3'), NV348b (5'-GRTTMACCCAAGAITCAAA-3') and NV348c 
Laboratory-confirmed norovirus infections by calendar week and year of notification, compared previous seasons, Germany, week 26 2016-week 22017 ( $n=56,384$ )

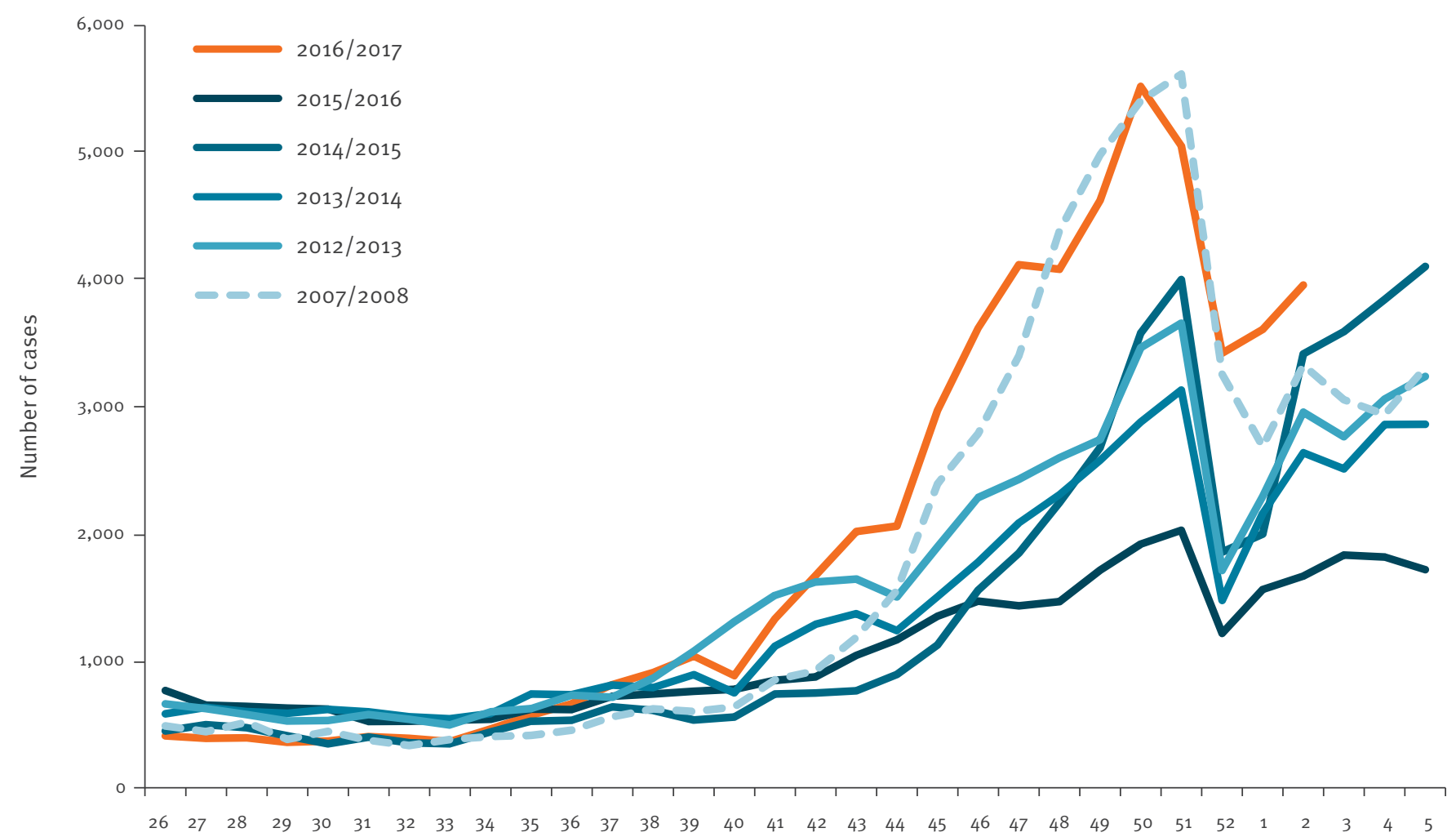

Calender week

Data as on 24 Jan 2017

(5'-GRTTRACCCAIACTTCAAA-3') for the first PCR (2328 bp fragment). The second PCR reaction was carried out using the HotStarTaq Master Mix Kit (Qiagen, Hildesheim, Germany) and primers NV6 (5'-TACCACTATGATGCAGATTA-3'), NV6a (5'-TATCACTATGATGCTGACTA-3'), NV348a, NV348b, $\mathrm{NV}$ 348c. PCR conditions were $5 \mathrm{~min}$ at $55^{\circ} \mathrm{C}, 55 \mathrm{~min}$ at $45^{\circ} \mathrm{C}, 2 \mathrm{~min}$ at $94^{\circ} \mathrm{C}$, followed by 40 cycles of $15 \mathrm{~S}$ at $94^{\circ} \mathrm{C}, 30 \mathrm{~s}$ at $45^{\circ} \mathrm{C}, 3 \mathrm{~min}$ at $68^{\circ} \mathrm{C}$ and finally $5 \mathrm{~min}$ at $68^{\circ} \mathrm{C}$. The resulting $2,274 \mathrm{bp}$ amplicons were subjected to direct sequencing. Nucleotide sequences of these samples were submitted to the GenBank database with the accession numbers $\mathrm{KY}_{357449}$ to $\mathrm{KY}_{357462 \text {. }}$

\section{Molecular genetic results}

We identified emerging recombinant norovirus strains previously not described in Germany in outbreaks or in sporadic cases of AGE. Typing results of all 240 analysed samples are shown in the Table.

The phylogenetic analysis revealed a recombination of Gll.P16 (ORF1) and Gll.2 (ORF2) strains (Figures 2 and 3). Using SimPlot analysis, the recombination point could be mapped to the ORF1/ORF2 junction region at nucleotide positions $732-734$ (data not shown). The recombinant strain GII.P16-GII.2 was detected in 29 of 69 investigated outbreaks, in nine of the 11 federal states of Germany that had outbreaks (Baden-Wuerttemberg, Bavaria, Berlin, Hesse, Lower Saxony, Mecklenburg-Western Pomerania, North Rhine-Westphalia, Rhineland-Palatinate and Thuringia) and was considered as the aetiological agents in 31 of 65 cases of sporadic AGE. The new recombinant was detected in specimens obtained from the sporadic cases in four hospitals in Berlin, North RhineWestphalia, Baden-Wuerttemberg and Lower Saxony. Besides the new recombinant strain, the well-known norovirus genotypes GI.P3-GI.3 and GII.P17-GII.17 and the recombinant strains GII.Pe-GII.4 2012 and GII.P4 2009-GIl.4 2012 were found co-circulating, were but less frequently detected in the current season.

\section{Discussion}

We found a new norovirus strain GII.P16-GIl.2 in samples from sporadic AGE and from norovirus outbreaks derived from nine federal states of Germany. It was recently shown that the emergence of new GII.4 norovirus variants can result in an increasing number of reported norovirus infection [5]. This has already been observed in Germany in the season 2007/08 which was 


\section{FIGURE 2}

Phylogenetic analysis based on the nucleotide sequence of a 357 bp region (ORF1) of genogroup II norovirus, Germany, 2016/17 ( $n=14$ representative samples)

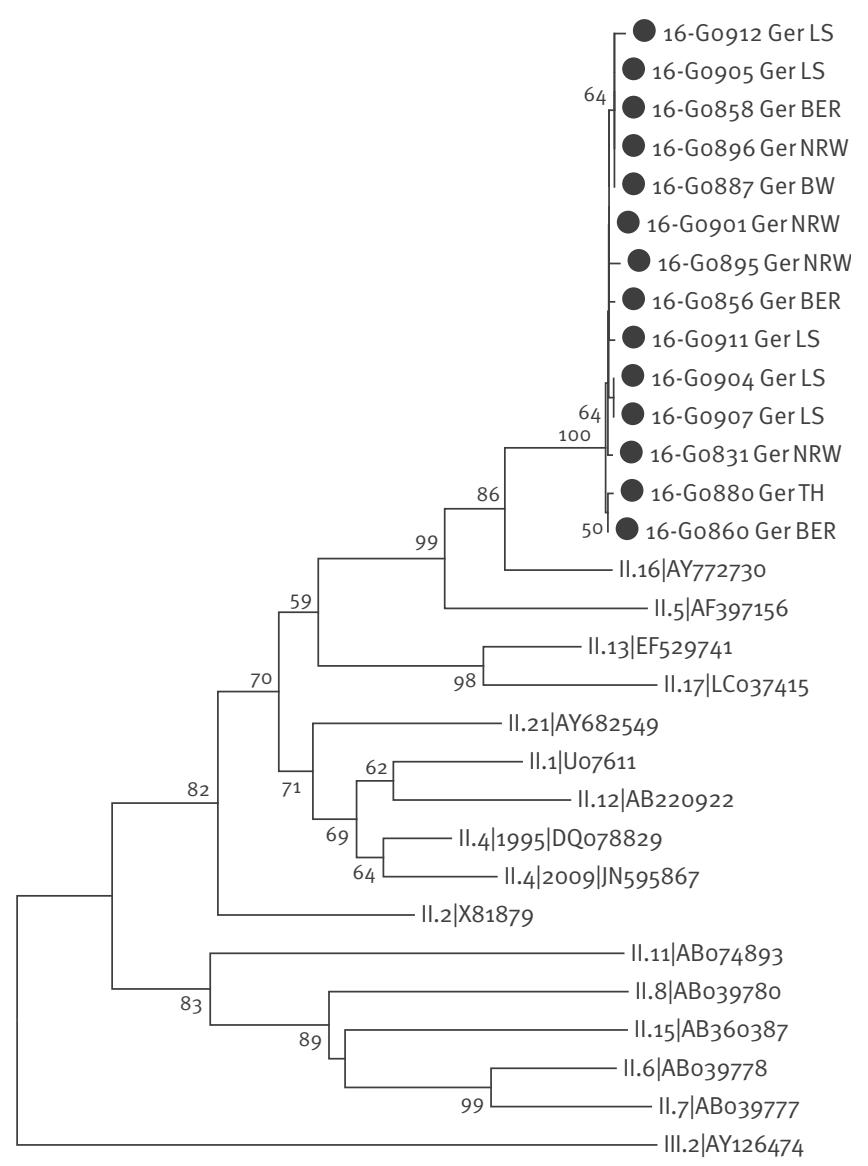

$\longmapsto$

BER: Berlin; BW: Baden-Wuerttemberg; Ger: Germany; LS: Lower Saxony; NRW: North Rhine-Westphalia; TH: Thuringia.

Analysed sequence: nucleotide positions 4,332-4,689 according to accession no: AY772730. Strains analysed in this study are denoted with a black bullet $(\bullet)$. Scale bar indicates nucleotide substitution per site. Sequence alignments were performed with the ClustalW algorithm. Neighbour-joining phylogenetic tree was produced using the MEGA 7 software with bootstrap test ( 1,000 replicates). Bootstrap values ( 1,000 replicates) above 50 are shown. The evolutionary distances were computed using the Kimura-2 parameter method.

also characterised by an early rise and high total number of notified norovirus infections, with most of the analysed outbreaks caused by the new epidemic variant GII.4 2006b [7]. Another example is the emergence of a novel variant GII.P17-GII.17 in the season 2014/15, which was first genotyped in China and Japan and replaced the previously dominant genotype GII.Pe-GII.4 2012 with an increased outbreak activity $[8,9]$. The new 2016 GII.P16-GIl.2 recombinant has sporadically been reported to the international molecular surveillance database NoroNet from Australia, Finland, France and Russia, and previously from Japan and China $[10,11]$, suggesting a worldwide distribution.

\section{FIGURE 3}

Phylogenetic analysis based on the nucleotide sequence of a 628 bp region of the P2 domain (ORF2) of genogroup II noroviruses, Germany, 2016/17 $(n=14$ representative samples)

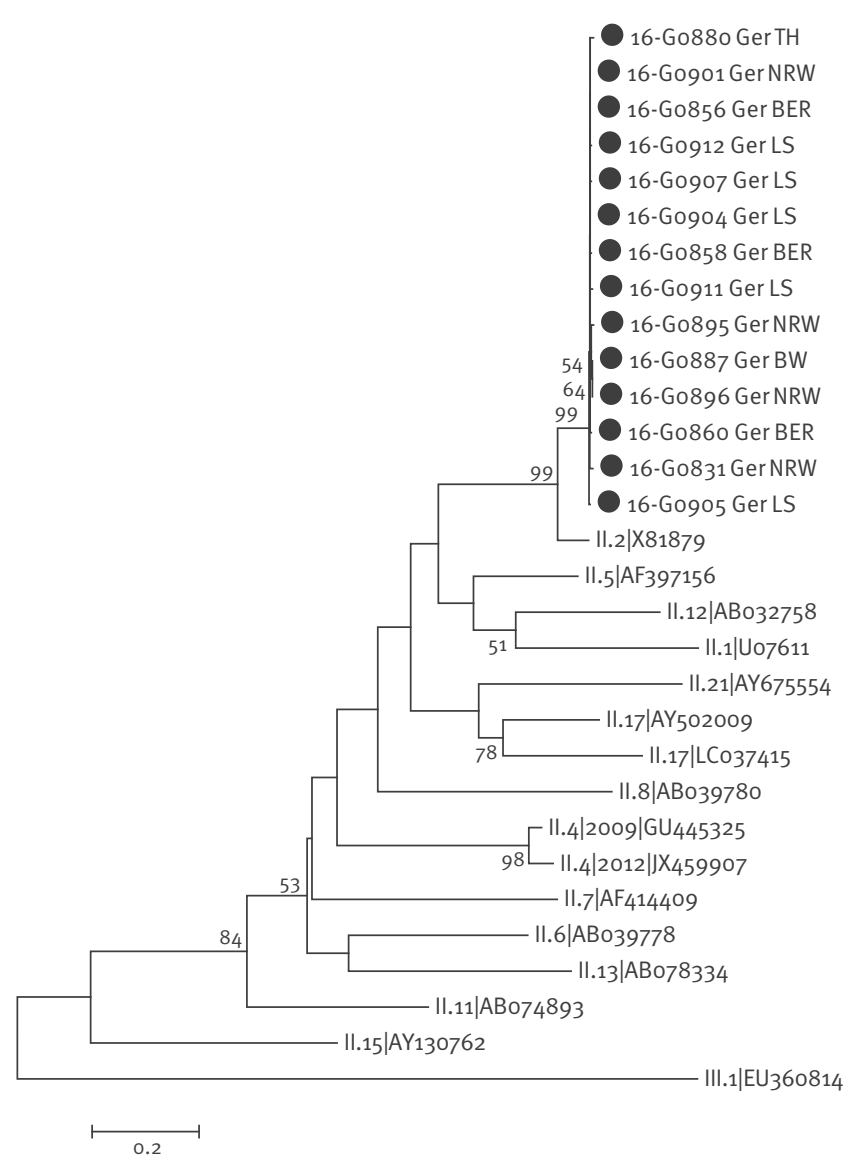

BER: Berlin; BW: Baden-Wuerttemberg; Ger: Germany; LS: Lower Saxony; NRW: North Rhine-Westphalia; TH: Thuringia.

Analysed sequence: nucleotide positions 5,871-6,509 according to accession no: AY772730. Strains analysed in this study are denoted with a black bullet $(\bullet)$. Scale bar indicates nucleotide substitution per site. Sequence alignments were performed with the ClustalW algorithm. Neighbour-joining phylogenetic tree was produced using the MEGA 7 software with bootstrap test $(1,000$ replicates). Bootstrap values ( 1,000 replicates) above 50 are shown. The evolutionary distances were computed using the Kimura-2 parameter method.

So far, it is unclear whether the new recombinant is associated with more severe symptoms. Further molecular and epidemiological investigations are needed to assess whether the emerging new recombinant norovirus strain GII.P16-GIl.2 can replace the predominant GII.Pe-GII.4 2012 strain and how this will affect outbreak sizes, course of disease and herd immunity of the population, not only in Germany but also in other countries in Europe. 


\section{TABLE}

Distribution of norovirus genotypes detected in samples sent to the Consultant Laboratory (outbreaks vs. sporadic cases of acute gastroenteritis), Germany, SeptemberDecember 2016 ( $\mathrm{n}=65$ samples from sporadic cases, $\mathrm{n}=175$ samples from 69 outbreaks)

\begin{tabular}{|l|l|l|l|l|}
\hline \multirow{2}{*}{ Norovirus genotype } & \multicolumn{2}{|l|}{ Sporadic cases } & \multicolumn{2}{l|}{ Outbreaks } \\
\cline { 2 - 5 } & $n$ & $\%$ & $n$ & $\%$ \\
\hline GI.P1-GI.1 & 0 & 0 & 1 & 1.4 \\
\hline GI.P3-GI.3 & 3 & 4.6 & 2 & 2.9 \\
\hline GI.P4-GI.4 & 1 & 1.5 & 3 & 4.3 \\
\hline GI.P5-GI.5 & 0 & 0 & 1 & 1.4 \\
\hline GI.Pb-GI.6 & 0 & 0 & 2 & 2.9 \\
\hline GII.P2-GII.2 & 1 & 1.5 & 0 & 0 \\
\hline GII.P4 2009-GII.4 2012 & 5 & 7.7 & 7 & 10.1 \\
\hline GII.P7-GII.6 & 1 & 1.5 & 4 & 5.8 \\
\hline GII.P7-GII.7 & 0 & 0 & 3 & 4.3 \\
\hline GII.P8-GII.8 & 0 & 0 & 1 & 1.4 \\
\hline GII.P16-GII.2 & 31 & 47.7 & 29 & 42.0 \\
\hline GII.P16-GII.4 2012 & 7 & 10.8 & 7 & 10.1 \\
\hline GII.P17-GII.17 & 0 & 0 & 6 & 8.7 \\
\hline GII.P21-GII.3 & 2 & 3.1 & 1 & 1.4 \\
\hline GII.P21-GII.13 & 1 & 1.5 & 0 & 0 \\
\hline GII.Pe-GII.4 2012 & 12 & 18.5 & 2 & 2.9 \\
\hline GII.Pg-GII.1 & 1 & 1.5 & 0 & 0 \\
\hline Total & 65 & 100 & 69 & 100 \\
\hline
\end{tabular}

\section{Acknowledgements}

We thank the staff of local public health authorities for collecting samples and data and Kathrin Stanossek, Roswitha Lorenz, Sonja Zimmermann and Ute Obst for excellent technical support.

\section{Conflict of interests}

None declared.

\section{Authors' contributions}

Wrote the paper: SN, MF. Analysed the data: SN, SJ, MF. Collected samples and data: AMEH, JH, OZ. Critical review the manuscript: SJ, MF, CTB, MH. Conceived and designed the experiments: $\mathrm{SN}, \mathrm{MH}$.
4. Bull RA, Eden JS, Rawlinson WD, White PA. Rapid evolution of pandemic noroviruses of the Gll.4 lineage.PLoS Pathog. 2010;6(3):e1000831. DOI: 10.1371/journal.ppat.1000831 PMID: 20360972

5. Bruggink L, Catton M, Marshall J. A norovirus intervariant GII.4 recombinant in Victoria, Australia, June 2016: the next epidemic variant?Euro Surveill. 2016;21(39):30353. DOI: 10.2807/1560-7917.ES.2016.21.39.30353 PMID: 27719750

6. Höhne M, Niendorf S, Mas Marques A, Bock CT. Use of sequence analysis of the $\mathrm{P}_{2}$ domain for characterization of norovirus strains causing a large multistate outbreak of norovirus gastroenteritis in Germany 2012.Int J Med Microbiol. 2015;305(7):612-8. DOI: 10.1016/j.ijmm.2015.08.010 PMID: 26341330

7. Bernard $\mathrm{H}$, Höhne $M$, Niendorf S, Altmann D, Stark K. Epidemiology of norovirus gastroenteritis in Germany 20012009: eight seasons of routine surveillance. Epidemiol Infect. 2013;142(1):63-74. DOI: 10.1017/S0950268813000435 PMID: 23517686

8. Han J, Ji L, Shen Y, Wu X, Xu D, Chen L. Emergence and predominance of norovirus Gll.17 in Huzhou, China, 2014-2015. Virol J. 2015;12(1):139. DOI: 10.1186/s12985-015-0370-9 PMID: 26362650

9. Matsushima Y, Ishikawa M, Shimizu T, Komane A, Kasuo S, Shinohara M, et al. Genetic analyses of GII.17 norovirus strains in diarrheal disease outbreaks from December 2014 to March 2015 in Japan reveal a novel polymerase sequence and amino acid substitutions in the capsid region. Euro Surveill. 2015;20(26):21173. DOI: 10.2807/1560-7917. ES2015.20.26.21173 PMID: 26159307

10. Iritani N, Kaida A, Abe N, Kubo H, Sekiguchi J, Yamamoto $\mathrm{SP}$, et al. Detection and genetic characterization of human enteric viruses in oyster-associated gastroenteritis outbreaks between 2001 and 2012 in Osaka City, Japan. J Med Virol. 2014;86(12):2019-25. DOI: 10.1002/jmv.23883 PMID: 24415518

11. Wang YH, Zhou DJ, Zhou X, Yang T, Ghosh S, Pang BB, et al. Molecular epidemiology of noroviruses in children and adults with acute gastroenteritis in Wuhan, China, 2007-2010. Arch Virol. 2012;157(12):2417-24. DOI: 10.1007/s00705-012-1437-1 PMID: 22886184

\section{License and copyright}

This is an open-access article distributed under the terms of the Creative Commons Attribution (CC BY 4.0) Licence. You may share and adapt the material, but must give appropriate credit to the source, provide a link to the licence, and indicate if changes were made.

This article is copyright of the authors, 2017.

\section{References}

1. Ahmed SM, Hall AJ, Robinson AE, Verhoef L, Premkumar P, Parashar UD, et al. Global prevalence of norovirus in cases of gastroenteritis: a systematic review and meta-analysis. Lancet Infect Dis. 2014;14(8):725-30. DOI: 10.1016/S14733099(14)70767-4 PMID: 24981041

2. Kroneman A, Vega E, Vennema H, Vinjé J, White PA, Hansman $\mathrm{G}$, et al. Proposal for a unified norovirus nomenclature and genotyping. Arch Virol. 2013;158(10):2059-68. DOI: 10.1007/ S00705-013-1708-5 PMID: 23615870

3. de Graaf M, van Beek J, Vennema H, Podkolzin AT, Hewitt J, Bucardo F, et al. Emergence of a novel GIl.17 norovirus End of the GIl.4 era? Euro Surveill. 2015;20(26):21178. DOI: 10.2807/1560-7917.ES2015.20.26.21178 PMID: 26159308 